\title{
CONTROLE DE BOBINAS DE HELMHOLTZ COM O USO DE FONTE DE CORRENTE CONTROLADA POR TENSÃO, CONTROLADOR PID DIGITAL E FILTRAGEM DE SINAL COM MÍNIMOS QUADRADOS EM LOTES
}

\author{
DANilo A. OLIVEIRA ${ }^{1}$, VALDEMiR CARRARA ${ }^{1}$, RiCARDO T. CARVALHO $^{2}$.
}

1. Departamento de Mecânica Espacial e Controle, Instituto Nacional de Pesquisas Espaciais Av. dos Astronautas, 1758, São José dos Campos, SP, Brazil, Caixa Postal: 515, CEP 12227-010 E-mails: danilo_dasors@yahoo.com.br, val@dem.inpe.br

\author{
2. Divisão de Física Aplicada, Instituto de Estudos Avançados \\ Rodovia dos Tamoios km 5,São José dos Campos, SP, Brazil, CEP 12228-840 \\ E-mail:ricc_33@yahoo.com.br
}

\begin{abstract}
The use of Helmholtz coils to generate controlled magnetic fields requires an effective system, with precision and stability, to control the electric current, supplying the needs of field generation. Aiming to supply these needs, in this work was developed a current control system, based on operational amplifiers and digital PID controller, where the controller acts reducing the difference between the requested field and the generated field to zero, even in the presence of disorders not predictable. Since the environment in which the coils are located may undergo electromagnetic interference and magnetometer measurements can be noisy, the method of Least Squares Lots on data obtained by the magnetometer was applied, eliminating these noises and increasing the efficiency of the controller. Therefore, was possible to generate controlled fields with variation less than $1.5 \mathrm{mG}$ even on presence of disturbs, with maximum standard deviation $\sigma= \pm 0.49 \mathrm{mG}$.
\end{abstract}

Keywords_- Helmholtz Coil, Current Supply, Least Squares

Resumo- O uso de bobinas de Helmholtz para a geração de campos magnéticos controlados necessita de um sistema de controle de corrente elétrica eficaz, com precisão e estabilidade, atendendo as necessidades de geração de campo. Visando atender estas necessidades, foi desenvolvido um sistema de controle de corrente realimentado, baseado em amplificadores operacionais e um controlador PID digital, onde o controlador age de maneira a reduzir a diferença entre o campo solicitado e o campo gerado a zero, mesmo na presença distúrbios não previsíveis. Uma vez que o ambiente em que as bobinas estão localizadas possa sofrer interferência eletromagnética e as medidas do magnetômetro possam ser ruidosas, foi aplicado o método de Mínimos Quadrados em Lotes nos dados obtidos pelo magnetômetro eliminando parte desses ruídos e aumentando a eficiência do controlador. Como resultado foi possível gerar campos controlados com variação menor que $1,5 \mathrm{mG}$ mesmo na presença de distúrbios, com desvio padrão máximo de $\sigma= \pm 0,49 \mathrm{mG}$.

Palavras-chave— Bobinas de Helmholtz, Fonte de Corrente, Mínimos Quadrados

\section{Introdução}

Bobinas de Helmholtz, empregadas em situações que requerem a geração de campos magnéticos controlados, encontram vasta aplicação em instrumentação, calibração de sensores, testes de equipamentos, sistemas de desmagnetização e simulação de sistemas embarcados de controle de atitude e validação de algoritmos de simulação. Robert (2003) cita experimentos com bobinas de Helmholtz circulares e enfatiza sua aplicabilidade, a facilidade e o custo de fabricação. Vale ressaltar que as bobinas de Helmholtz podem variar suas características, como formato e dimensão, dependendo das aplicações e projetos em que serão empregadas.

Para o controle do campo magnético no interior do conjunto de bobinas, este trabalho apresenta um sistema capaz de simular o campo magnético terrestre empregando um controlador PID digital e uma fonte de corrente controlada por tensão. $\mathrm{O}$ conjunto de bobinas de Helmholtz na qual foi feito o controle possui o formato cúbico, com comprimento de um metro e 3 eixos ortogonais (Figura 1), e o projeto mecânico está descrito em Carrara, V. (2010)

Stupak (1995) apresenta um método para a modelagem do campo a partir da equação de BiotSavart, obtendo uma constante que relaciona o campo gerado com a corrente elétrica aplicada nas bobinas. $\mathrm{O}$ uso de um controlador PID digital elimina a necessidade de cálculo dessa constante, e ajusta gradativamente os valores da corrente aplicada no conjunto de bobinas até que o erro entre o campo solicitado e o campo gerado seja igual a zero. Para o controle da corrente elétrica no conjunto de bobinas, projetou-se uma eletrônica baseada em amplificadores operacionais, capaz de fornecer uma corrente elétrica estabilizada por meio de um controlador PI analógico.

Como a realimentação da malha do controlador PID digital utiliza a telemetria de um magnetômetro posicionado no interior do conjunto de bobinas, aplicou-se o método de Mínimos Quadrados em Lotes para filtrar os dados obtidos pelo magnetômetro, eliminando-se parte dos ruídos e aumentando a eficiência do controlador. Shirai (2003) mostra a influência de campos magnéticos externos e ruídos em conjunto de bobinas de Helmholtz e demonstra que campos 
magnéticos estáveis podem ser gerados usando um sistema de compensação automática.

Nos próximos capítulos serão descritos o projeto eletrônico da fonte de corrente, o sistema de filtragem de dados do magnetômetro, o programa de controle e acionamento do conjunto de bobinas e quais foram os resultados obtidos.

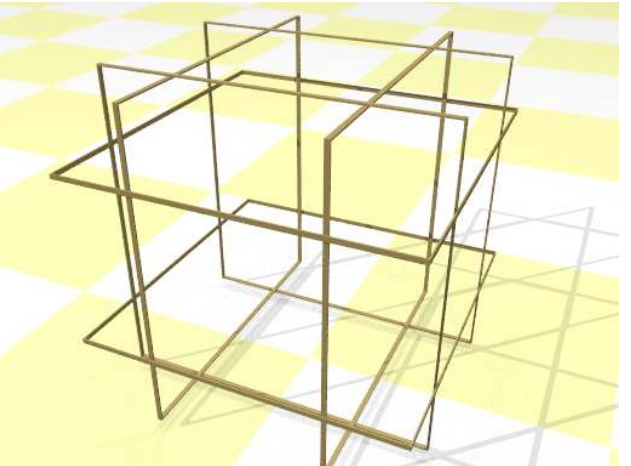

Figura 1. Conjunto de bobinas de Helmholtz com formato cúbico

\section{Fonte de Corrente Controlada por Tensão}

Para o acionamento das bobinas foi desenvolvido uma eletrônica que fornece a corrente elétrica necessária para a geração do campo magnético solicitado a partir de uma tensão de referência, e para cada par de bobinas, alinhadas sobre os eixos $X, Y$ e $Z$. O sistema de controle de corrente realimentado apresenta uma relação de comparação entre o sinal de referência $\left(V_{s p}\right)$ e a saída (tensão em um resistor sensor $\left.V_{\text {sensor }}\right)$, cuja diferença é utilizada como sinal de erro na malha de controle PI. O sistema tenta agir de maneira a reduzir a diferença, ou sinal de erro $\xi$, a zero, mesmo na presença distúrbios (Ogata, K., 2007). Este tipo de controlador é usado principalmente em sistemas onde não é conhecido o modelo da planta, e portanto, métodos analíticos não podem ser utilizados.

A tensão de referência $V_{s p}$ é obtida a partir da filtragem de um sinal de PWM. O PWM por sua vez, é gerado por um microcontrolador 8051 , e passa por um multiplexador que ajusta a amplitude do sinal em 5V. Em seguida passa por um filtro passa-baixa, e então é inserido no controlador PI. A saída do controlador aciona a base do Mosfet ligado em série com o conjunto de bobinas (Figura 2), controlando assim a corrente que passa por elas. Um resistor-sensor, com resistência de $0,1 \Omega$, é ligado em série com a saída do Mosfet, pois a partir da tensão medida sobre o resistor é possível obter a corrente elétrica que flui pela bobina. O diagrama do processo de acionamento pode ser visto na Figura 3. Antes de ser realimentada no controlador analógico, a tensão no sensor é amplificada com um ganho $A$ por um amplificador operacional.

Se o erro do controle for nulo, então $V_{s p}=A V_{\text {sen- }}$ sor, e o valor da corrente seguindo lei de Ohm e é dada por

$$
I=\frac{V_{s p}}{A R_{\text {sensor }}},
$$

onde $R_{\text {sensor }}$ é a resistência do resistor sensor, $A$ é o ganho, e $V_{s p}$ é a tensão de referência.

No controlador PI mostrado na Figura 2, tem-se que os ganhos $K_{i}=1 /\left(R_{14} C_{4}\right)$ e $K_{p}=R_{12} / R_{13}$. Dada a entrada $V_{s p}$ no controlador e a saída $V_{m o s}$, que aciona a base do Moesfet, a função de transferência do controlador é dada por

$$
\frac{V_{m o s}(s)}{V_{s p}(s)}=\frac{R_{12} R_{14} C_{4} s+R_{13}}{R_{14} R_{13} C_{4} s} .
$$

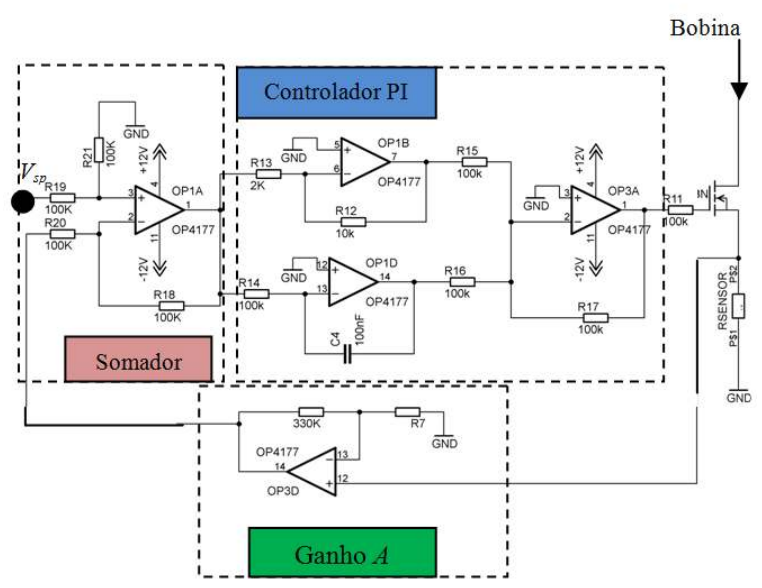

Figura 2. Diagrama da fonte de corrente controlada por tensão

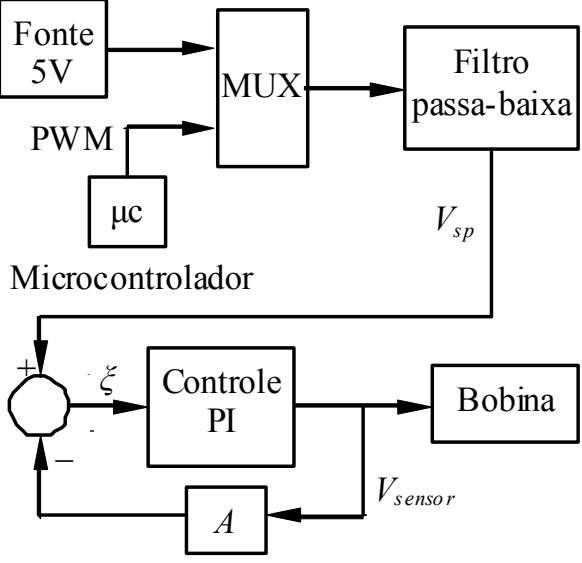

Figura 3. Diagrama de blocos do processo de ajuste de corrente nas bobinas

Como a bobina é ligada em série com o resistor sensor $\left(R_{\text {sensor }}\right)$, como visto na Figura 2, e considerando que o Mosfet possui características de acionamento linear, para a entrada $V_{\text {mos }}$ e a saída $V_{\text {sensor, }}$, tem-se que a função de transferência para essa ligação em série (bobina-resistor) é dada por

$$
\frac{V_{\text {sensor }}(s)}{V_{\text {mos }}(s)}=\frac{R_{\text {sensor }}}{L s+R_{\text {sensor }}} .
$$

A função de transferência para o sistema completo em malha aberta é dado por

$$
\frac{V_{\text {sensor }}(s)}{V_{\text {mos }}(s)}=\frac{R_{\text {sensor }} R_{12} R_{14} C_{4} s+R_{13} R_{\text {sensor }}}{L R_{14} R_{13} C_{4} s^{2}+R_{13} R_{14} C_{4} R_{\text {sensor }}}
$$


O diagrama de blocos para o sistema completo,incluindo o ganho $A$ da realimentação, é apresentado na Figura 4.

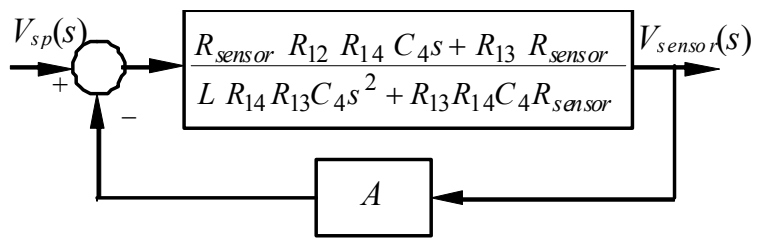

Figura 4. Diagrama da função de transferência do sistema

Definindo os valores de $R_{14}=100 \mathrm{k} \Omega, \mathrm{C}_{4}=100$ $\mathrm{nF}, R_{12}=10 \mathrm{k} \Omega$ e $R_{13}=2 \mathrm{k} \Omega$, de maneira que $K_{i}=100 \mathrm{e}$ $K_{p}=5$, e o ganho $A=1$, a resposta do sistema aplicando um degrau unitário é totalmente estável e não passa por regiões de instabilidade, como mostram as Figuras 5 e 6.

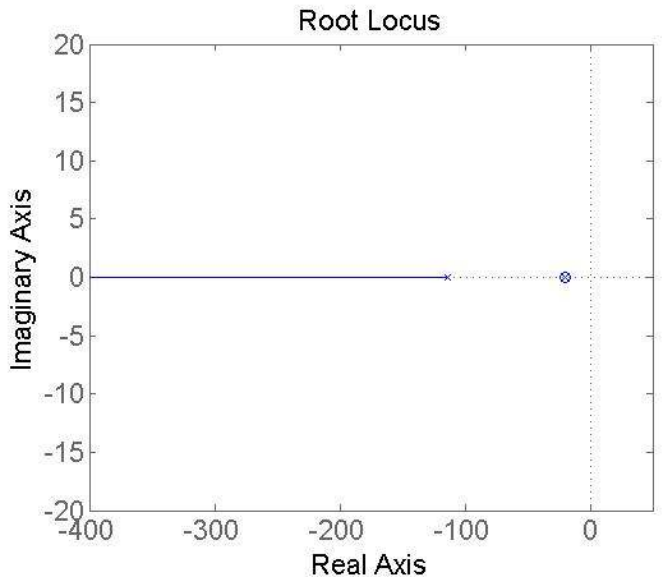

Figura 5. Lugar das raízes do sistema

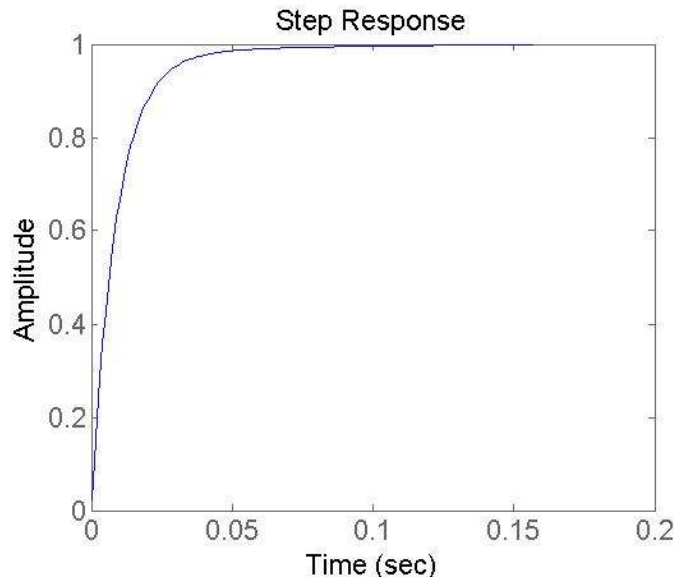

Figura 6. Resposta do sistema dado um degrau unitário

\section{Filtragem dos dados do magnetômetro}

Como a realimentação da malha de controle utiliza a telemetria de um magnetômetro posicionado no interior do conjunto de bobinas, e estes dados estão sujeitos a ruídos, foi aplicado o Método dos Mínimos
Quadrados para filtrar o sinal e ter melhor aproveitamento do controlador. O Método dos Mínimos Quadrados é uma técnica que procura encontrar o melhor ajuste para um conjunto de dados tentando minimizar os resíduos (Maybeck, P.S., 1979). Uma maneira de processar os dados é uso do Mínimo Quadrados em Lotes (MQL), onde a equação clássica é dada por

$$
\hat{\mathbf{x}}=\left(\mathbf{H}^{t} \mathbf{W} \mathbf{H}\right)^{-1} \mathbf{H}^{t} \mathbf{W} \mathbf{y},
$$

sendo y o vetor que contém $m$ medidas do campo magnético, $\mathbf{x}$ o vetor os parâmetros a serem estimados, $\mathbf{H}$ a matriz que relaciona as medidas aos parâmetros (número de medidas) e $\mathbf{W}$ é a matriz que pondera os diferentes tipos de erros, incluindo os de medida do magnetômetro. A matriz $\mathbf{W}$ é dada por

$$
\mathbf{W}=\left[\begin{array}{cccc}
\frac{1}{\sigma_{1}^{2}} & 0 & \cdots & 0 \\
0 & \frac{1}{\sigma_{2}^{2}} & \cdots & 0 \\
\vdots & 0 & \ddots & 0 \\
0 & 0 & \cdots & \frac{1}{\sigma_{m}^{2}}
\end{array}\right]
$$

onde $\sigma$ é o desvio padrão da medida.

Neste caso definiu-se o desvio padrão da medida $\sigma=1$, e, dado um conjunto de dez medidas do campo magnético $(m=10)$, o filtro atua de maneira a traçar uma reta $B=a m+b$ utilizando os parâmetros estimados $(a$ e $b)$ e retorna o valor no ponto médio dessa reta, sendo

$$
B=a\left(\frac{m}{2}\right)+b \text {. }
$$

Espera-se que o valor de $a$ seja o mais próximo de zero, mas caso o valor de $a$ seja significativo, utilizou-se o ponto médio $m / 2$ para melhor aproximação do valor esperado.

O magnetômetro utilizado para a leitura do campo magnético foi o VMFS-51 (Vectronic, 2010) onde teste realizado mostrou que, para a leitura do campo magnético terrestre atuando sobre o eixo $X$ das bobinas, houve um desvio padrão de $0,47 \mathrm{mG}$ para os valores obtidos com o magnetômetro (Figura 7), enquanto após passar pelo filtro, esse valor foi para $0,17 \mathrm{mG}$ (Figura 8 ).

\section{Programa de Controle}

Para acionar o conjunto de bobinas, desenvolveu-se um programa de controle onde usuário informa as componentes do campo magnético a ser gerado, e um controlador PID digital atua de maneira a reduzir a diferença entre campo magnético solicitado e o campo magnético gerado à zero, utilizando os dados enviados pelo magnetômetro. O controlador PID altera diretamente os valores a serem carregados nos registradores que geram os sinais PWMs, e esses 
valores são enviados e armazenados no microcontrolador a cada iteração.

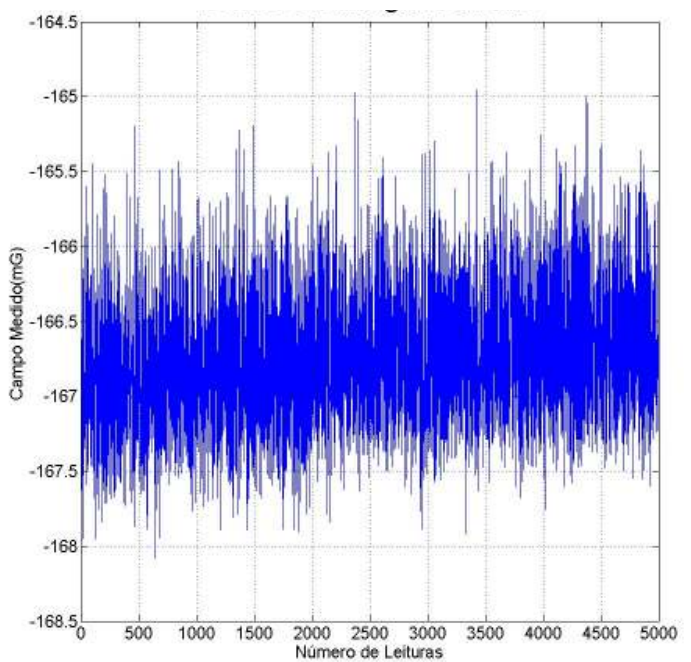

Figura 7. Leitura do campo magnético terrestre que passa alinhado sobre o eixo $X$ do conjunto de bobinas realizada pelo magnetômetro

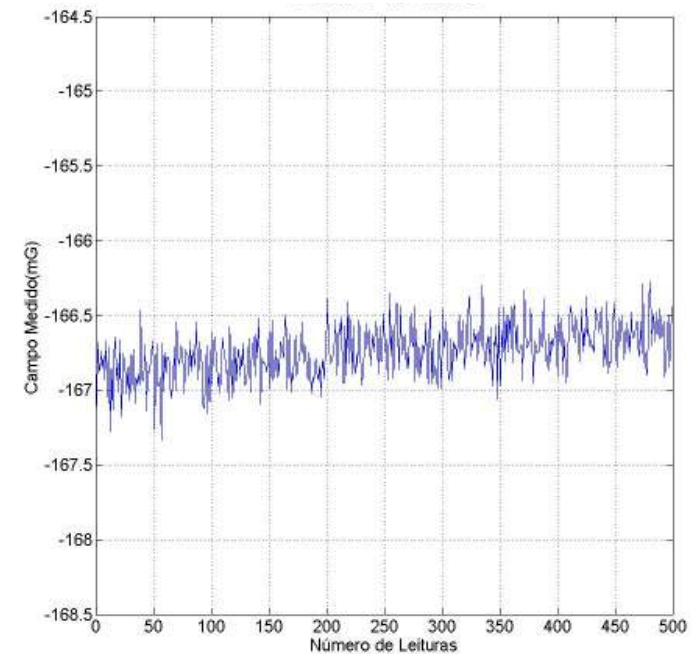

Figura 8. Leitura do campo magnético terrestre que passa alinhado sobre o eixo $X$ do conjunto de bobinas após passar pelo filtro MQL

Com os valores carregados nos registradores, o microcontrolador gera o sinal de referência e envia à fonte de corrente que alimenta o conjunto de bobinas. As informações que realimentam o controlador PID digital são as medidas do campo magnético realizada pelo magnetômetro posicionado no interior do conjunto de bobinas, após passar pelo filtro MQL. Esse processo ocorre simultaneamente nos 3 eixos do conjunto de bobinas (Figura 9), e para este controlador PID digital, os ganhos foram ajustados sendo $K_{p}=$ $50, K_{i}=20$ e $K_{d}=10$.

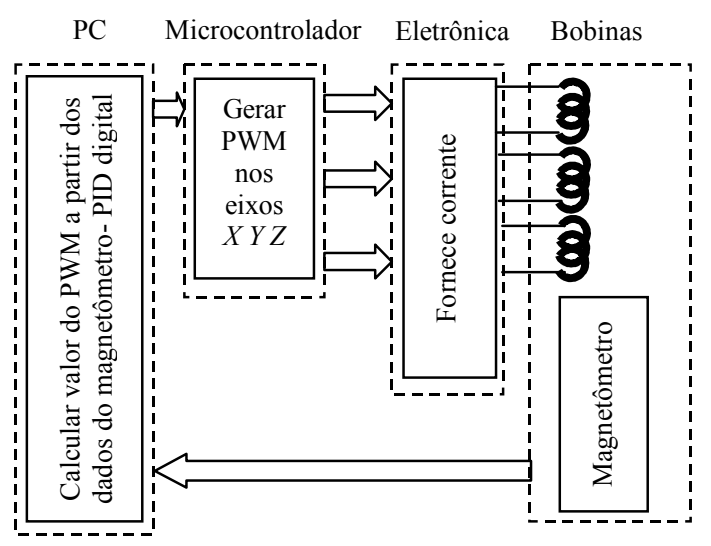

Figura 9. Processo de controle do campo magnético no conjunto de bobinas

\section{Resultados Obtidos}

Como teste de operacionalidade, foi solicitado a geração de um campo magnético nulo $(0,0,0) \mathrm{mG}$ no interior do conjunto de bobinas. A resposta do sistema de controle nos eixos $X, Y$ e $Z$ podem ser observadas nas Figuras 10, 12 e 12. No eixo $X$, em regime permanente, o campo foi mantido estável com variação menor $1 \mathrm{mG}$ mesmo na presença de ruídos, com um desvio padrão de $0,29 \mathrm{mG}$. No eixo $Y$ a variação também foi menor que $1 \mathrm{mG}$, sendo o desvio padrão de $0,29 \mathrm{mG}$. No eixo $Z$ a variação ficou abaixo de $1,5 \mathrm{mG}$, apresentando um desvio padrão de $0,49 \mathrm{mG}$.
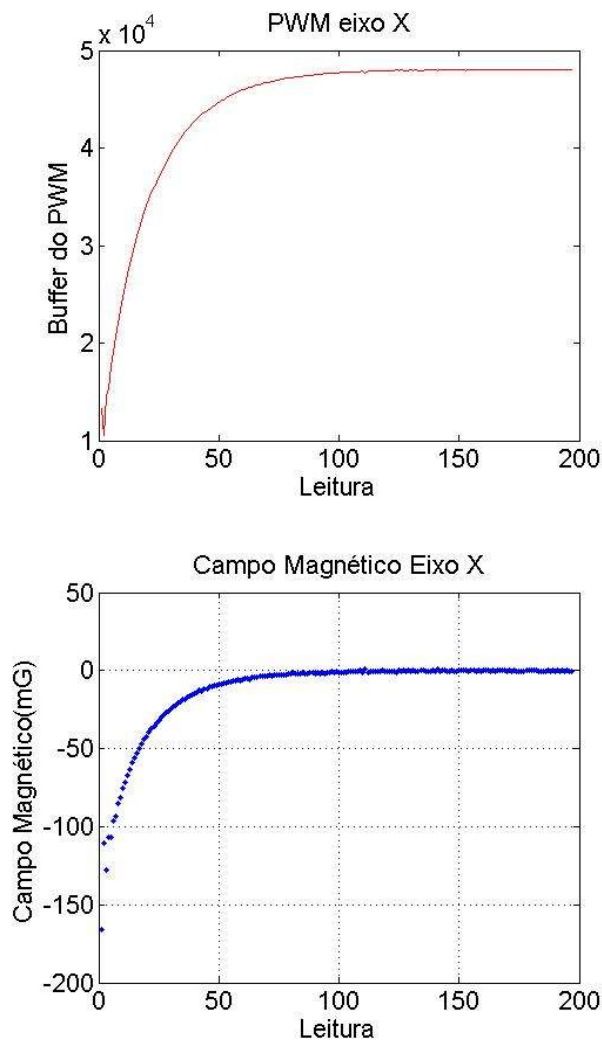

Figura 10. a- Valor do sinal PWM aplicado nas bobinas alinhadas sobre o eixo $X$. b-Resposta do campo magnético gerado sobre o eixo $X$ do conjunto de bobinas quando solicitado um campo nulo 

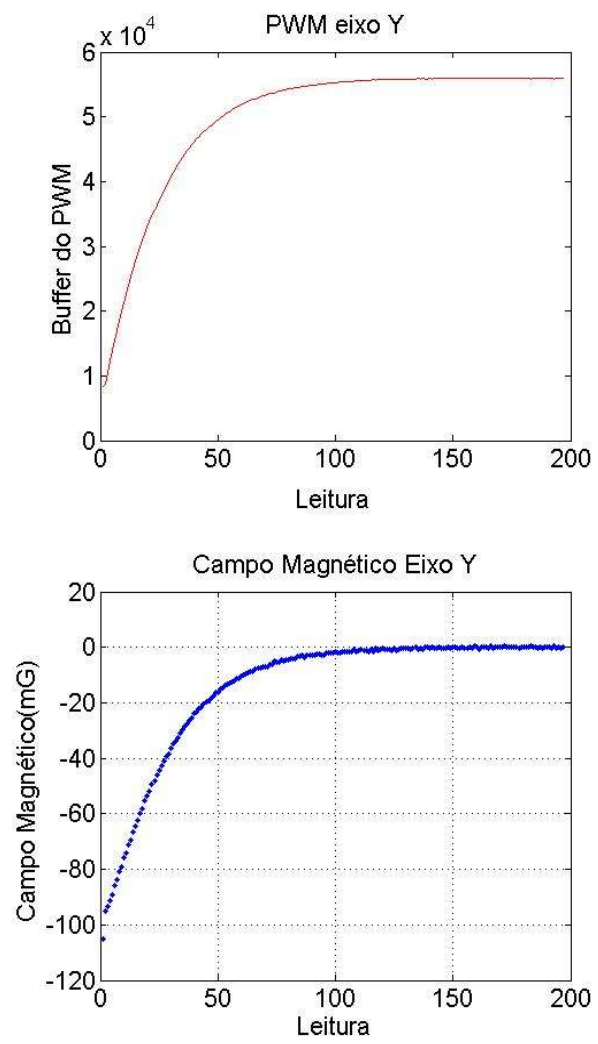

Figura 11. a- Valor do sinal PWM aplicado nas bobinas alinhadas sobre o eixo $Y$. b-Resposta do campo magnético gerado sobre o eixo $Y$ do conjunto de bobinas quando solicitado um campo nulo
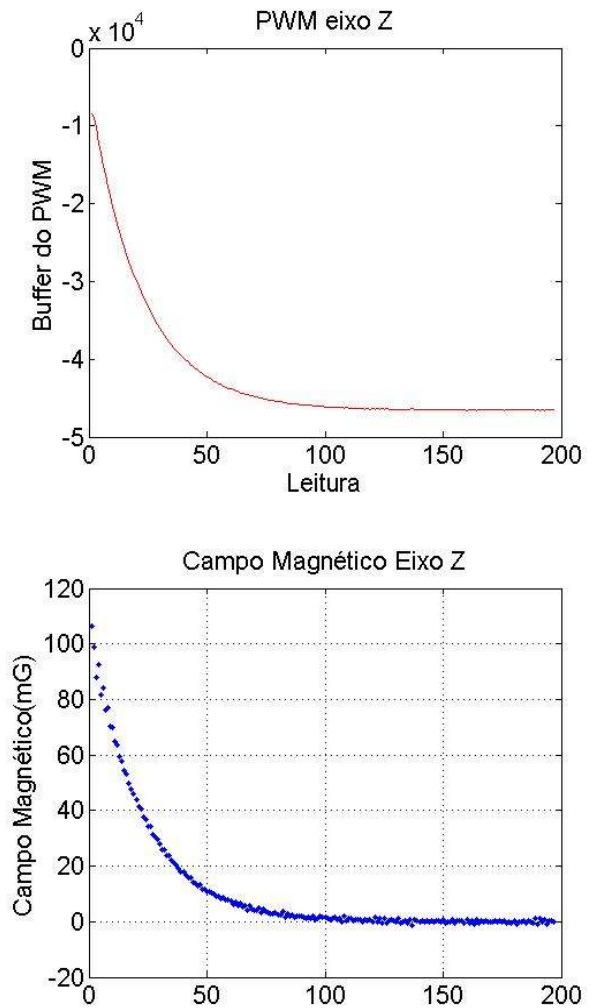

Figura 12. a- Valor do sinal PWM aplicado nas bobinas alinhadas sobre o eixo $X$. b-Resposta do campo magnético gerado sobre o eixo $Z$ do conjunto de bobinas quando solicitado um campo nulo

\section{Conclusões}

Neste trabalho foi apresentado um método de controle de campo magnético em bobinas de Helmholtz com 3 eixos ortogonais utilizando uma fonte de corrente controlada por tensão e um controle PID digital. Como as medidas realizadas pelo magnetômetro apresentam ruídos, gerando queda na eficiência do controlador, foi aplicado um filtro MQL nos dados obtidos pelo magnetômetro. Durante a leitura do campo magnético terrestre que passa alinhado ao eixo $X$ do conjunto de bobinas, o desvio padrão era de $0,47 \mathrm{mG}$ para os valores obtidos com o magnetômetro, enquanto após a aplicação do filtro o desvio padrão caiu para $0,17 \mathrm{mG}$.

O sistema de controle atuou de maneira que, quando solicitado a geração de um campo magnético nulo $(0,0,0) \mathrm{mG}$ no interior do conjunto de bobinas, $\mathrm{o}$ controlador manteve variação abaixo de $1,5 \mathrm{mG}$ em regime permanente, mesmo na presença de ruídos. No eixo $X$, o campo foi mantido estável com variação menor $1 \mathrm{mG}$ mesmo na presença de ruídos, com um desvio padrão de $0,29 \mathrm{mG}$. No eixo $Y$ a variação ficou abaixo de $1 \mathrm{mG}$, sendo o desvio padrão de $0,29 \mathrm{mG}$. No eixo $Z$ a variação foi menor que $1,5 \mathrm{mG}$, apresentando um desvio padrão de $0,49 \mathrm{mG}$.

\section{Referência Bibliográficas}

ROBERT, R.. Bobina de Helmholtz. Revista Brasileira de Ensino de Física, 2003, vol. 25, p. 4044. DOI: $10.1590 / \mathrm{S} 0102-47442003000100005$

CARRARA, V.. Projeto de bobinas magnéticas para uso em satélites. Ouro Preto: 2th Brazilian Conference on Eletromagnetics, 1996.

STUPAK, J. J.. A Method of Calibrating Helmholtz Coils for the Measurement of Permanent Magnets. Tualatin: IMCSD 24th Annual Proceedings, 1995.

SHIRAI, T.. A Magnetic Field Generator with Active Compensation of External Field. Measurement Science and Technology, 2004. p. 248-253.

DOI: 10.1088/0957-0233/15/1/036

MAYBECK, P.S.. Stochastic models, estimation and control. London: Academic Press, 1979p.

OGATA, K.. Engenharia de Controle Moderno. São Paulo: Prentice-Hall, 2007. 788p.

VECTRONIC. Datasheet Magnetic Field Sensor Type VMFS-51. Vectronic Aerospace GmbH: 2010. Disponível em http://www.vectronicaerospace.com/files/VAS-VMFS51-DS2.pdf. Acesso em 16 maio 2013. 